This is a postprint version of the following published document:

Oliva, Antonio de la, et al. Xhaul: toward an integrated fronthaul/backhaul architecture in $5 \mathrm{G}$ networks. IEEE Wireless Communications, 22(5), pp. 3240, October 2015

DOI: $\underline{\text { https://doi.org/10.1109/MWC.2015.7306535 }}$

(C) 2015 IEEE. Personal use of this material is permitted. Permission from IEEE must be obtained for all other uses, in any current or future media, including reprinting/republishing this material for advertising or promotional purposes, creating new collective works, for resale or redistribution to servers or lists, or reuse of any copyrighted component of this work in other works. 


\section{XHAUL: TOWARD AN INTEGRATED FRONTHAUL/BACKHAUL ARCHITECTURE IN $5 G$ NETWORKS}

\section{Antonio de la Oliva, Xavier Costa Pérez, Arturo Azcorra, Andrea Di Giglio, Fabio CaValiere, Dirk TiegelbekKers, JohanNes lessmanN, Thomas Haustein, Alain MOURAD, AND PAOLA IOVANNA}

Antonio de la Oliva is with University Carlos III of Madrid.

Xavier Costa Pérez and Johannes Lessmann are with NEC Europe Ltd, Germany.

Arturo Azcorra is with University Carlos III of Madrid and IMDEA Networks.

Andrea Di Giglio is with Telecom Italia.

Fabio Cavaliere and Paola Iovanna are with Ericsson Research, Italy.

Dirk Tiegelbekkers is with Nokia Solutions and Networks.

Thomas Haustein is with Fraunhofer Heinrich Hertz Institute.

Alain Mourad is with InterDigital Europe Ltd.

\section{ABSTRACT}

The Xhaul architecture presented in this article is aimed at developing a $5 \mathrm{G}$ integrated backhaul and fronthaul transport network enabling flexible and software-defined reconfiguration of all networking elements in a multi-tenant and service-oriented unified management environment. The Xhaul transport network vision consists of high-capacity switches and heterogeneous transmission links (e.g., fiber or wireless optics, high-capacity copper, mmWave) interconnecting remote radio heads, $5 \mathrm{G}$ points of attachment (5GPoAs, e.g., macro- and small cells), centralized-processing units (mini data centers), and points of presence of the core networks of one or multiple service provider(s). This transport network shall flexibly interconnect distributed $5 \mathrm{G}$ radio access and core network functions, hosted on network centralized nodes, through the implementation of a control infrastructure using a unified, abstract network model for control plane integration (Xhaul Control Infrastructure, XCI); and a unified data plane encompassing innovative high-capacity transmission technologies and novel deterministic-latency switch architectures (Xhaul packet Forwarding Element, XFE). Standardization is expected to play a major role in a future $5 \mathrm{G}$ integrated fronthaul/backhaul architecture for multi-vendor interoperability reasons. To this end, we review the major relevant activities in the current standardization landscape and the potential impact on the Xhaul architecture.

\section{The Need For a Novel Integrated BACKHAUL/FroNTHAUL NETWORK}

According to recent predictions [1], mobile data traffic will increase 11-fold between 2013 and 2018 . Fifth generation $(5 \mathrm{G})$ radio access network (RAN) technologies serving this mobile data tsunami will require fronthaul and backhaul solutions between the RAN and packet core to deal with this increased traffic load. Furthermore, there will be a sizeable growth in the capillarity of the network since traffic load increase in the $5 \mathrm{G}$ RAN is expected to stem from an increased number of base stations with reduced coverage (i.e., mobile network densification).

To support the increased density of the mobile network (e.g., in terms of interference coordination) and achieve the required $5 \mathrm{G}$ capacity, extensive support for novel air interface technologies such as cooperative multipoint (CoMP), carrier aggregation (CA), and massive multiple-input multiple-output (MIMO) [2] will be needed. Such technologies require processing of information from multiple base stations simultaneously at a common centralized entity and also tight synchronization of different radio sites. Hence, backhaul and fronthaul will have to meet more stringent requirements not only in terms of data rate but also in terms of latency, jitter, and bit error rate [3].

Given that the aforementioned challenges will be addressed by service providers in a business environment where revenues do not increase proportionally to the data volume, costeffective network deployment, efficient operation, and evolution strategy are required. A promising approach to address this challenge, as can be observed in major standardization bodies, such as the European Telecommunications Standards Institute (ETSI) network functions virtualization (NFV), is virtualization, which exploits the multiplexing gain of softwarized network functions on top of commoditized hardware. This has led to the centralized RAN $[4,5]$ concept where cellular base station functions are hosted in cloud computing centers. Once virtualized, base station functions can be flexibly distributed and moved across data centers, providing another degree of freedom for load balancing. Moreover, base station functions can be decomposed in many different ways [6], giv- 
ing rise to the so-called flexible functional split, where the radio protocol split between centralized and distributed base station functions can be adjusted on a case-by-case basis.

In this context, the distinction between fronthaul and backhaul transport networks will blur as varying portions of functionality of $5 \mathrm{G}$ points of attachment (5GPoAs) might be moved toward the network as required for cost efficiency reasons. The traditional capacity overprovisioning approach on the transport infrastructure will no longer be possible with 5G. Hence, a new generation of integrated fronthaul and backhaul technologies will be needed to bring capital expenditure (CAPEX) and operational expenditure (OPEX) to a reasonable return on investment (ROI) range. Also, for cost reasons, the heterogeneity of transport network equipment must be tackled by unifying data, control, and management planes across all technologies as much as possible. A redesign of the fronthaul/ backhaul network segment is a key point for $5 \mathrm{G}$ networks since current transport networks cannot cope with the amount of bandwidth required for 5G. Next generation radio interfaces, using 100 $\mathrm{MHz}$ channels and squeezing the bit-per-megahertz ratio through massive MIMO or even fullduplex radios, requires a 10 -fold increase in capacity on the Xhaul segment, which cannot be achieved just through the evolution of current technologies. Considering the new challenges brought by $5 \mathrm{G}$, this article presents a novel architecture aimed at integrating fronthaul and backhaul in a common packet-based network defined as Xhaul. This work is relevant due to the increased interest in architectures integrating both the fronthaul and backhaul network segments, currently seen as one of the major bottlenecks of the network. The presented architecture is in line with the ideas of recently created standardization bodies, such as the Next Generation Fronthaul Interface Alliance (NGFI) or the work performed in IEEE 1904.3 and IEEE 802.1. The remainder of this work is structured as follows. We present the proposed Xhaul architecture with the key characteristic of integration of fronthaul and backhaul network segments into a common packet-based framework. Following that, we present the key challenges that must be addressed for Xhaul to become a reality. These challenges will require modifications to key standards and the development of new ones; hence, we provide a brief overview of the expected standardization impact. The article concludes with a summary.

\section{XhaUl Architecture}

To address the aforementioned challenges, we propose the Xhaul architecture, aimed at developing the next generation of $5 \mathrm{G}$ integrated backhaul and fronthaul networks enabling a flexible and software-defined reconfiguration of all networking elements in a multi-tenant and service-oriented unified management environment. The envisioned Xhaul transport network will consist of high-capacity switches and heterogeneous transmission links (e.g., fiber or wireless optics, high-capacity copper, or millimeter-wave) interconnecting remote radio heads, 5GPoAs (e.g., macro and small cells), pooled-processing units (mini data centers), and points of presence (PoPs) of the core networks of one or multiple service providers.

This requires completely new physical layer technologies or a radical evolution of existing ones, such that the challenging $5 \mathrm{G}$ performance requirements can be met. The Xhaul architecture will use a novel unified data plane protocol able to transport both backhaul and fronthaul traffic, regardless of the functional RAN split. As an example, major challenges for such a protocol are the big amount of data to handle, the synchronization of user data, and reflection of the channel structure of RAN protocols, such as Third Generation Partnership Project (3GPP) Long Term Evolution (LTE) dedicated traffic channel (DTCH, logical channel), downlink shared channel (DL-SCH, transport channel), and physical downlink shared channel (PDSCH, physical channel) [7]. Thereby, the information granularity of the RAN protocol should be maintained (e.g., bearer and user specific information), and complemented by the necessary routing information.

Furthermore, the designed unified data plane protocol must allow for future RAN evolution, which may happen on shorter timescales than transport network upgrades. The new data plane will also need new switch architectures to support the required latency and jitter demands. Hence, significant effort is needed for the combined development of transmission technologies, switch architectures, and protocols in a way that allows CPRI/OBSAI ([8,9]) data to be transported in a packetized form over the unified Xhaul data plane.

It is worth highlighting that full centralization might not always be the solution for a particular 5GPoA. In particular, outdoor small cells can be extremely difficult to connect to the network, as they are usually mounted at the street level instead of the rooftop level, and many different obstacles (buildings, trees, etc.) might obstruct the line of sight (LOS) to a transport network point of presence. Hence, in some cases, nonLOS technologies can be the only option to provide small cell connectivity in meshed scenarios. While non-LOS technologies are typically far from reaching the capacities required for RAN centralization, they will still be considered for the Xhaul architecture given their relevance within the RAN ecosystem.

Finally, the novel architecture will also require the development of a unified control and management plane (network model and interface primitives) to simplify network operations across heterogeneous technologies. It is understood that the Xhaul architecture must be able to coexist with legacy infrastructure and must allow operators smooth migration. The key idea of the proposed architecture is to move from a traditional transport network perspective to a novel architecture integrating both fronthaul and backhaul into the Xhaul transport network.

The two key elements of the Xhaul architecture are explained in the following and shown in Fig. 1.

The Xhaul packet forwarding element (XFE): This element is the central part of the Xhaul infrastructure, integrating the different physical technologies used for fronthaul and backhaul through a common data frame and forwarding
To address these challenges, we propose the Xhaul architecture, aimed at developing the next generation of $5 \mathrm{G}$ integrated backhoul and fronthaul networks enabling a flexible and software-defined reconfiguration of all networking elements in a multi-tenant and serviceoriented unified management environment. 


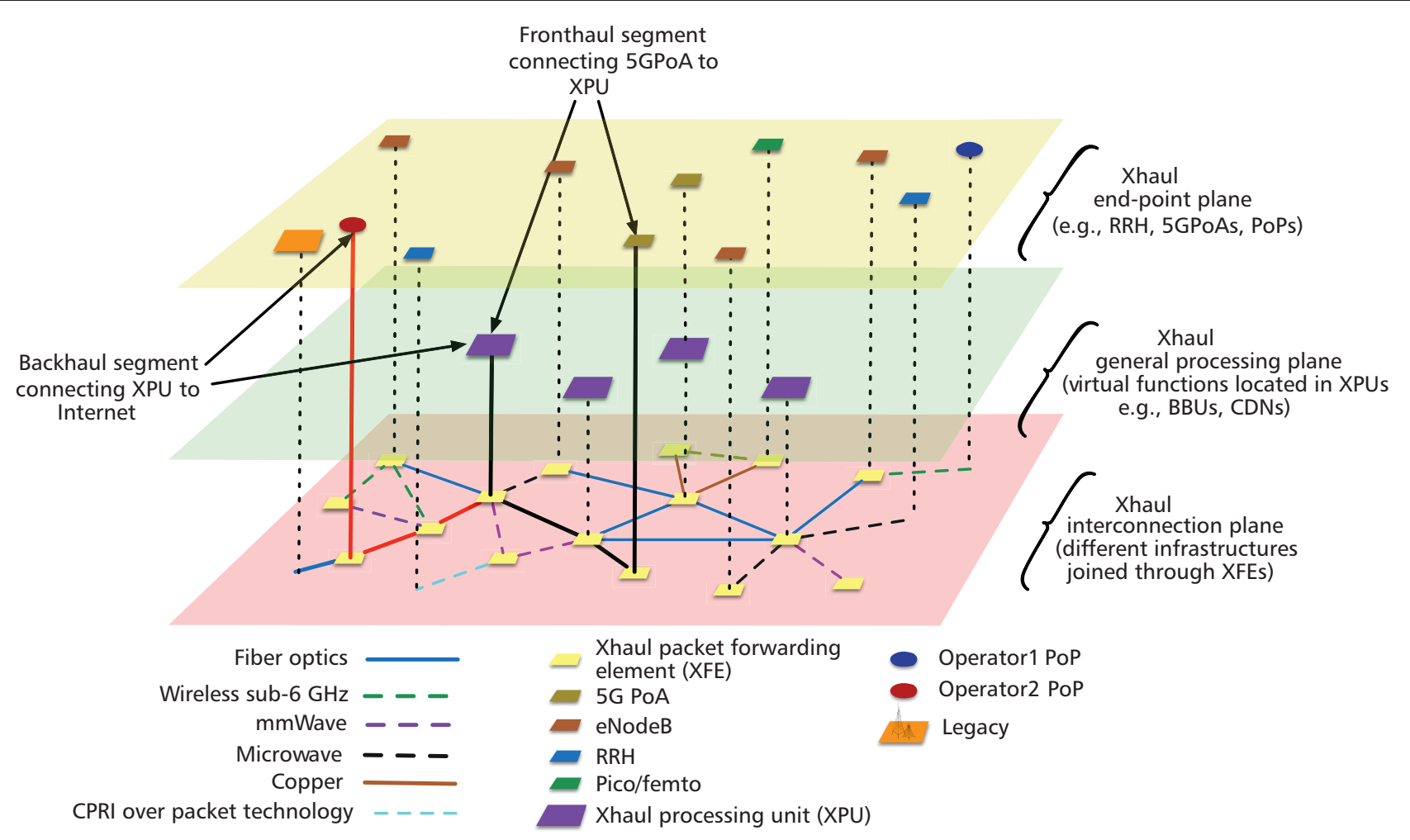

Figure 1. Xhaul physical infrastructure.

behavior. Developing a flexible frame format is a key aspect of fronthaul/backhaul integration, allowing the transport of fronthaul/backhaul traffic on the same physical link, replacing different technologies by a uniform transport technology for both network segments.

The Xhaul control infrastructure (XCI): This element is a control infrastructure using a unified abstract network model for control plane integration. The XCI will be developed by extending existing software defined network (SDN) controllers to provide the services for novel northbound and southbound interfaces (NBI and SBI), and enable multi-tenancy support in trusted environments. The key aspect of the XCI is the development of new mechanisms to abstract the mobile transport network and aggregate measured contextual information.

Figure 1 shows the physical infrastructure of which Xhaul is composed, categorized into three differentiated layers. The bottom layer corresponds to the interconnection plane and shows the networking infrastructure, formed by heterogeneous links connecting the different elements of the planes located above. Xhaul will typically connect an element of the end-point plane (uppermost plane in Fig. 1) with an element in the general processing plane (middle plane in Fig. 1). This represents the interconnection of, say, a remote radio head implementing radio sampling with a virtualized baseband unit (BBU).

The interconnection plane makes use of Xhaul packet forwarding elements (XFEs) to interconnect a broad set of novel technologies to create a packet-based network that can meet the demands of $5 \mathrm{G}$ networks. The technologies, which have already been identified as relevant for the future of backhauling, are represented in the figure too. They span from fiber optics to novel CPRI-overpacket technologies, also considering wireless links such as millimeter-wave (mmWave).

The second plane (depicted in green in Fig. 1) has been named the Xhaul general processing plane and shows the different Xhaul processing units (XPUs) that carry out the bulk of the operations in Xhaul. The functionality provided by these XPUs is multi-faceted. It highly depends on the actual interconnection and must encompass functionality expected from the different elements in the $5 \mathrm{G}$ network (depicted in the uppermost layer of Fig. 1). As an example, in (partially) centralized RAN implementations, the XPUs will host BBUs or medium access control (MAC) processors (thus enabling true cloud RAN). Furthermore, they might serve as end-points for services or even caches. The different functional distributions between 5GPoA and XPU, and the different services that can be hosted in the XPUs are one of the pillars of the flexibility provided by the Xhaul architecture. This is represented by the different connection options between the uppermost (end-point plane) and the middle layer of Fig. 1.

Figure 2 shows the Xhaul functional structure and the new Xhaul services. The lowest layer corresponds to the overlay of all infrastructure layers in Fig. 1. The middle layer represents one of the key concepts associated with Xhaul: the integration of the different technologies (including fronthaul and backhaul) in a common packet network based on technology abstraction, unified framing and common data, and the control and management planes. Finally, the upper layer presents a selection of the features envisioned for the Xhaul infrastructure: 


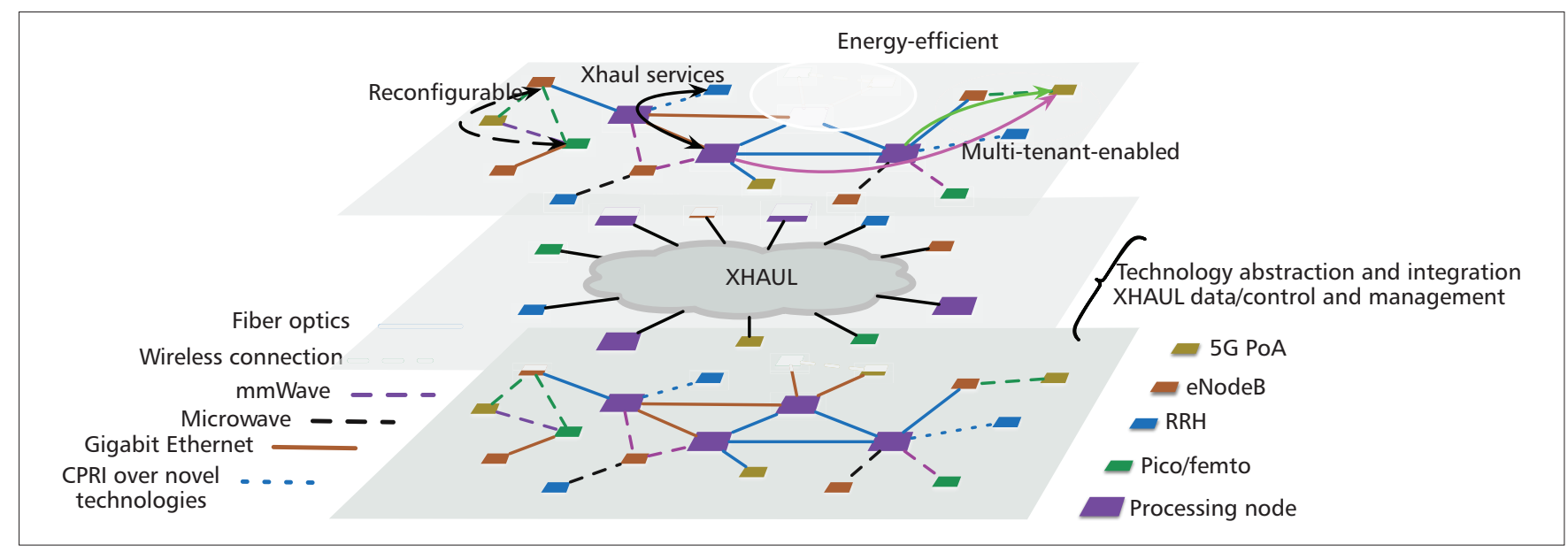

Figure 2. Xhaul functional structure.

- Reconfigurability: To cope with the level of demand expected from 5GPoAs, the Xhaul must be able to allocate capacity in a dynamic way, reallocating resources from areas where they are not needed to meet the demands in busier areas (e.g., joint RAN and Xhaul capacity optimization).

- Energy efficiency: This is achieved through techniques to reduce the energy consumption of the different Xhaul elements, combined with the joint optimization of RAN and Xhaul resources in terms of dynamic de-activation or decommissioning of scarcely used network portions.

- Multi-tenant operation: To enable generalized sharing and more efficient utilization of the underlying resources, Xhaul will implement the concept of multi-tenancy at the infrastructure level. Moreover, Xhaul allows operators and over-the-top (OTT) companies to quickly deploy services within the Xhaul platform such as video on demand, HD videoconferencing, TV broadcasting, content delivery networks (CDNs), and cloud services.

The overall Xhaul concept envisioned is depicted in Fig. 2. Xhaul will integrate the current functions of backhaul and fronthaul with all the technologies unified by the use of a common framing. The data path of the Xhaul will be handled by the XFE (on OSI layer 2), interconnecting different heterogeneous technologies.

The control and management planes of the network will be handled by a set of controllers (XCIs) interacting with the data plane through a novel SBI capable of supporting the multiple technologies available in the Xhaul physical layer. The XCI not only controls packet forwarding (i.e., the XFE behavior) but also the physical layer parameters (including optical equipment). The XCI will implement a powerful and open NBI to support a variety of network applications running dedicated optimization algorithms and functions for specific Xhaul features, such as capacity reconfiguration, forwarding, and infrastructure on demand (IoD) features.

\section{Key R\&D Challenges}

This section is devoted to analyzing the key technical challenges to be solved in order to make the Xhaul architecture a reality.

\section{NOVEL TECHNOLOGIES FOR Ultra-High Capacity Transport Networks}

In order to cope with the requirements of $5 \mathrm{G}$ it is necessary to identify the technologies enabling the Xhaul network, and understand how these technologies can be combined to realize integrated backhaul and fronthaul switching nodes, known as Xhaul forwarding elements (XFEs). Another key aspect is making the different underlying technologies transparent to upper control layers according to the SDN paradigm. This poses several outstanding technical challenges. A first challenge is the definition of a unified mechanism for packet and constant bit rate transport, able to deal with the stringent requirements demanded by the fronthaul links in terms of bandwidth and bounded latency. The mechanism should support not only CPRI but be open to different split options of radio protocols between baseband and remote radio units, making the challenge even harder. The mechanism will rely on unified protocol framing for fronthaul and backhaul, and the combination of layer 1 (L1) and layer 2 (L2) switching for aggregation/disaggregation of multiple fronthaul flows and Ethernet backhaul traffic over such a universal transport container. A second aspect addressed is to define how existing technologies should be adapted to support the Xhaul concept and identify new technologies that should be introduced to fill any gap in terms of latency, bandwidth, or lack of proper infrastructure. For example, millimeter waves or wireless optics could be used when optical fiber or copper cables are not installed. When a fixed access network is in place, operators could find that it is convenient to reuse it for Xhaul purposes, but this is not trivial because it was typically designed for much less demanding applications. Finally, greenfield installation opens the door to completely new technologies, such as silicon photonics for optical switches, which could cut down the equipment cost on the order of magnitudes. It is clear from the previous discussion that a one-fits-all approach does not work for Xhaul, but different technologies apply to different use cases. This poses the problem of how to manage these technologies so that the operators are 
Regarding the unified

data frame, different

approaches need to be analysed to understand

the best way of unifying

the different require-

ments of the technolo-

gies. However, at this

time, using a modified

version of Ethernet

seems to be a promising

approach, since multiple

products already use the

Ethernet framing. unaware of any unnecessary technology details. To accomplish this task it is needed to design and develop the Xhaul SBI, especially developing the protocol and agent extensions to support the underlying Xhaul technologies. In the following we identify a set of potential technologies the development of which will highly impact the capacity of the Xhaul network segment.

New wireless transmission techniques, such as hybrid RF-optical wireless technologies [10], aimed at data rates as high as $10 \mathrm{~Gb} / \mathrm{s}$ over $1000 \mathrm{~m}$, as targeted in Xhaul. In outdoor environments wireless optics is sensitive to haze and fog, while mmWave faces challenges in rain, so a hybrid solution could be used to increase the link quality. The wireless optical solutions are robust to electromagnetic interference (EMI) and highly tap-proof, which makes them a good choice in security-aware environments. Wireless optics also have the key advantage of unlicensed optical spectrum.

Reconfigurable all-optical switches based on silicon photonics [11]. These devices enhance the flexibility of the Xhaul network with no significant additional cost, moving to optics the integration concepts well consolidated in electrical integrated circuits (ICs). This potentially leads to two orders of magnitude cost reduction with respect to conventional optical switches.

New flexible multiplexing and aggregation schemes, for example, based on flexi-grid and Flexi-passive optical network (PON). Digital signal processing (DSP)-enabled bandwidth-variable optical transceivers will be considered to interface access and Xhaul networks, based on multi-carrier (orthogonal frequency-division multiplexing, OFDM) technology for elastic modulation and multiplexing, introducing finer sub-wavelength granularity at the electrical level. This will result in fully programmable adaptive optical transceivers that can achieve optimal bandwidth allocation.

Techniques enabling massive and easy deployment of indoor small cells. More than 80 percent of wireless traffic is generated indoors, such as in offices, homes, and shopping malls [12]. Therefore, indoor capacity will be crucial for $5 \mathrm{G}$ success. However, this leads to a number of technical challenges: high penetration loss into buildings from the outdoor macro base stations, hostile RF propagation environment, and indoor RF regulations. Current solutions like distributed antenna systems (DASs) do not meet the $5 \mathrm{G}$ requirements for ultra-high capacity and density indoors, so new solutions are needed to: - Pool the baseband resources all over more buildings

- Introduce the capability of dynamic cell splitting to provide high capacity in hotspot areas and simultaneous mobility coverage in other areas

- Save energy consumption by switching off unused baseband resources, especially during closed hours of these buildings

- New bandwidth-efficient fronthaul solution, with reconfigurability features, to make such a design easy.

\section{Xhaul Unified Data Plane Design}

The Xhaul data plane has the ambitious objective of the design of a unified versatile frame format and the corresponding protocol suite, which supports:
- The traditional split of radio heads and 5GPoA functionality

- The concept of moving functionality of the 5GPoA among different XPUs

- The concurrent usage of fronthaul traffic (e.g., OBSAI/ CPRI over fiber) and backhaul traffic (e.g., Ethernet) on the same link

The new frame protocol suite will provide a common solution for different demands of bandwidth, latency, and synchronization caused by different split options of the 5GPoA and the different technologies available for the fronthaul/ backhaul. The main challenge of this task is the creation of a common data path that can simultaneously transport backhaul and fronthaul traffic with their different characteristics.

Regarding the unified data frame, different approaches need to be analyzed to understand the best way of unifying the different requirements of the technologies. However, at this time, using a modified version of Ethernet seems to be a promising approach, since multiple products already use the Ethernet framing. It is worth highlighting that traditional Ethernet switching cannot fulfill the stringent requirements on latency and jitter for fronthaul traffic [13], so substantial optimizations to the switching fabric will be required for the integration of fronthaul/backhaul. Ongoing initiatives (e.g., CPRI over Ethernet, time-sensitive networking [TSN]) also need to be analyzed and further developed for the new frame format to enable a cost-efficient and well accepted solution. Alternative options, like the optical transport network (OTN) defined in ITU-T G.709, or OTN-like framing protocols for the encapsulation and multiplexing of heterogeneous traffic data will also be considered. Currently, they present similar problems as Ethernet in dealing with stringent latency and jitter requirements.

\section{Xhaul Control Plane Design}

Another of the key challenges faced by the Xhaul architecture is the design of a common control and management plane: the Xhaul control infrastructure (XCI). The XCI will introduce an SDN-controlled packet-based network for the complete Xhaul network. One of the key features of the envisioned XCI is to enable the sharing of the Xhaul infrastructure by multiple operators with differentiated policy and trusted access to decrease the total cost of ownership (multi-tenancy). The XCI will also be designed in such a way as to promote the addition of new services to the transport network in a fast, simplified way. The XCI may be implemented through an SDN controller and its two main interfaces, the SBI and NBI. The SBI will provide technology abstraction and adaptation via a set of common control parameters; in this way the heterogeneous technologies forming the Xhaul data plane can be controlled and managed in a homogeneous way by using a common SDN driver to XCI. The XCI will provide another level of technology abstraction to the applications using the controller through the NBI.

The abstraction at the NBI level allows the SDN controller to deliver mechanisms to dynamically program the transport network. The XCI will collect common contextual information for 
traffic load, congestion, interference, and energy savings to retrieve an overall system status of the heterogeneous network. Metrics will be extrapolated by new methodologies from a large, versatile set of data. The metrics will be utilized by the Xhaul applications (including performance optimization of the network), which will form the basis of the resource orchestration.

The SBI allows common control and management of nodes as well as technology- and vendor-specific amendments. Several alternatives to the design of the XCI and the integration between XFE and XCI can be considered, such as application programming interface (API) calls, plug-ins, or a hierarchical concept of SDN controllers. In particular, the hierarchical SDN architecture proposed by the Open Network Foundation looks very promising as it introduces additional levels of scalability, modularity, reliability, and security, but it must not decrease the latency or increase the OPEX for the operators.

The key research challenge is to design the control mechanism of the XFEs in such a way that the different connection demands of fronthaul and backhaul can be provided by the same physical infrastructure. For example, the XCI needs to reserve sufficient bandwidth for fronthaul traffic with no latency and jitter degradation as well as configure the prioritization of the backhaul traffic in the XFEs. Applications of the XCI need to match the requirements of the different network elements to the available resources to establish a working network. Algorithms in XCI analyze the interaction between fronthaul and backhaul traffic and lighten the effort with an automated configuration approach. This matching has to follow topology changes due to network maintenance as well as dynamic changes of the network triggered by the XCI itself, such as shutting down small cells to preserve energy.

\section{Xhaul CONTEXT-AWARE RESOURCE ORCHESTRATION}

The resources forming the Xhaul network, such as physical nodes and links, are a major cost source in an operator's overall infrastructure. With the extreme RAN densification expected to come along with $5 \mathrm{G}$, total equipment cost is bound to increase even more, putting high pressure on operators' profitability. Furthermore, there are lots of dynamics that need to be addressed, such as short- and long-term traffic load fluctuations, time-dependent machine-type communication patterns, wireless channel variations on the access and transport side, and so on. Therefore, in order to maintain a reasonable cost-revenue balance, it is extremely important to provide measures that aim at optimally exploiting the existing network infrastructure at any point of time, avoiding overprovisioning. In the Xhaul context, such measures will get a new angle as resource management must be done with respect to the varying conditions of the wireless access, and the distribution (and redistribution) of base station and transport network functions across XPUs. Another factor coming into play will be energy efficiency (e.g., by shutting down base stations, dosing backhaul links, and remapping radio head nodes to BBU pools).
Compared to existing resource management schemes, Xhaul resource management needs much more stringent latency and jitter awareness, and much more fine-grained end-to-end quality of service (QoS) differentiation. Indeed, greater diversity of not only $5 \mathrm{G}$ user services, but also transport network services (packet-based phase/frequency/time synchronization, CPRI/OBSAI transport, etc.) must be accommodated. Additionally, the distribution of Xhaul functions not only depends on available node and link resources, but actually can have a profound impact on the service-level performance as well. For example, higher centralization of base station functions results in better RAN performance, while more decentralization reduces the burden on the transport network.

In summary, the key problem of resource management in Xhaul is the diversification and high number of variables to consider. The RAN may support different levels of centralization (fully or partially centralized, or fully distributed), different backhauling models (in-band, outof-band, licensed, point-to-point, point-tomultipoint), different physical technologies (line of sight, copper, fiber) and virtualized parts of the RAN and core networks may be located or even moved to different data centers depending on their load. This multitude of variables to consider is bounded by stringent requirements on delay and bandwidth, which affect the rest of the components due to the performance interrelation between RAN and fronthaul/backhaul. Due to this performance interrelation, the general problem of resource orchestration in Xhaul is the joint optimization of RAN policies (schedulers, shapers, handover, etc.), routing policies, and Xhaul function placement (e.g., BBU, virtual IP edge functions, mobility anchors, in-network processing functions). In the spirit of $5 \mathrm{G}$ ambitions, the Xhaul controller must interwork with controllers of the neighboring network domains (i.e., the RAN and core network controllers). Ultimately, the exact form of interaction will likely be via some global orchestration engine, with each domain controller (RAN, Xhaul, and core) in turn being controlled by that engine via a north-south interface.

\section{XHAUL ImPACT ON StandardS}

The Xhaul architecture's major enhancements and new capabilities have the potential for a significant impact on various standardization bodies. In the following we identify three standardization bodies' clusters relevant to the realization of the key Xhaul innovations.

Standardization cluster 1 - 3GPP, IEEE 802.11, SCF, BBF, NGMN:

- New functions and interfaces for both the data and control planes accounting for various functional splits between the core and RAN networks

- New underlying wired and wireless technologies that might require amendments to existing or new specifications for the backhaul, fronthaul, or access.

Standardization Cluster 2 - ITU-T, Photonics21, FSAN, ETSI, IEEE 1904:
In the spirit of 56 ambi-

tions, the Xhaul controller must interwork with controllers of the neighboring network domains (i.e., the RAN and core network controllers). Ultimately, the exact form of interaction will likely be via some global orchestration engine, with each domain controller in turn being controlled by that engine via a north-south interface. 
Next generation radio

access networks will be

characterized by a

higher degree of

capillarity, density and

higher bit rates. This will

push to the limit the

network segments

connecting the RAN to

the Core of the network,

the so called backhaul.

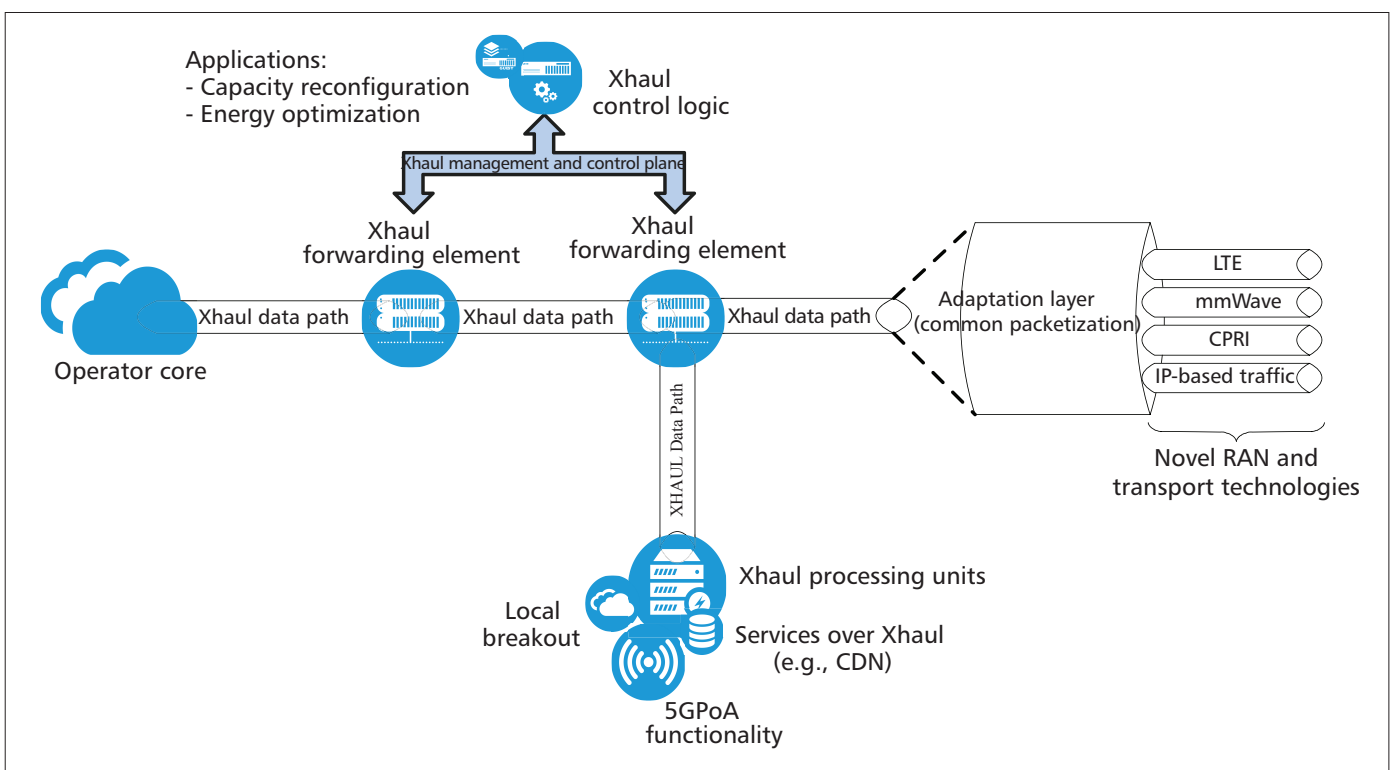

Figure 3. Xhaul concept design.

- Novel framing protocols for CPRI optical transport or innovative solutions for CPRI mapping over Ethernet, OTN, and wavelength-division multiplexing (WDM)

- New enabling technologies for optical fronthaul

Standardization Cluster 3 - ONF, IETF, ETSI, IEEE 802.1 OmniRAN, IEEE 802.21:

- Novel abstraction of the various underlying technologies providing the required awareness at the right level in the Xhaul system architecture

- Enhancements on the protocols and architectures of SDN and NFV-based systems, taking advantage of context awareness at various locations along the Xhaul path

Within these standardization clusters the relevant Xhaul activities per cluster are summarized in Table 1.

A cohesive and coordinated approach across the aforementioned standardization groups (siloed in nature) would then be desirable to maximize the Xhaul architecture benefits potential, as shown in Fig. 4.

\section{SUMmARY AND CONCLUSIONS}

Next generation RANs will be characterized by a higher degree of capillarity, density, and higher bit rates. This will push the network segments connecting the RAN to the core of the network, the so-called backhaul, to the limit. At the same time, new models for the design of base stations separating radio and baseband processing in different nodes separated geographically and connected by specific-purpose communication lines (fronthaul) are being deployed by operators as a way of decreasing the OPEX/CAPEX of their networks. With the increase in bandwidth required for $5 \mathrm{G}$, non-integrated fronthaul/backhaul architectures are not expected to be able to cope with the new capacity requirements in a cost-effective manner.

The Xhaul architecture proposed in this article is a $5 \mathrm{G}$ integrated backhaul and fronthaul transport network enabling flexible and software-defined reconfiguration of all networking elements in a multi-tenant and service-oriented unified management environment. Four key R\&D challenges have been identified to make the Xhaul architecture a reality:

- Novel technologies for ultra-high-capacity transport networks potentially leading to significant advantages in terms of connectivity, capacity and/or network simplification

- Xhaul unified data plane design of a unified, versatile frame format and the corresponding protocol suite supporting:

-The traditional split of radio heads and 5GPoA functionality

-The concept of moving functionality of the 5GPoA among different XPUs

-The concurrent usage of fronthaul traffic and backhaul traffic on the same link

- Xhaul control plane design of a common control and management plane, the Xhaul control infrastructure (XCI), introducing an SDNcontrolled packet-based network for the complete Xhaul network

- Xhaul context-aware resource orchestration considering the joint optimization of RAN policies, routing policies, and Xhaul function placement via a technology-abstracted SDN interface

Finally, we have reviewed the relevant standardization activities that could be impacted by the Xhaul architecture design and provided information regarding the specific groups to be targeted to enable the key Xhaul new functionality and features.

\section{ACKNOWLEDGMENTS}

The Xhaul architecture is a joint effort of 21 companies and academic institutions. The authors would like to thank the following persons (among many others who also participated) for their contribution to the ideas on this article: Chenguang Lu (Ericsson AB), Miguel Angel Puente (Atos Spain), Luis M. Contreras (Telefonica I +D), Anna Pizzinat (Orange), 
Third Generation Partnership Project (3GPP)

IEEE 802.11

Small Cell Forum (SCF)

Broadband Forum (BBF)

Next Generation Mobile Networks (NGMN)

SA1; SA2; SA5; RAN1; RAN2; RAN4.

HEW; NG60

Backhaul; Network; Radio \& Physical Layer; Services; Deployment

SIM-TD; E2E Architecture and Service Innovation

Small Cells; RAN evolution; ORI; 5 G initiative

\begin{tabular}{|c|c|}
\hline Standardization Cluster 2 & Activities \\
\hline $\begin{array}{l}\text { International Telecommunications Union - } \\
\text { Telecommunications Standardization Sector (ITU-T) }\end{array}$ & SG15 \\
\hline Photonics21 & WG1 Information and Communication \\
\hline Full Service Access Network (FSAN) & Next Generation PON (NG-PON) Task Group \\
\hline European Telecommunications & Open Radio Interface (ORI) ISG \\
\hline Standards Institute (ETSI) & \\
\hline IEEE 1904 & 1904 RoE \\
\hline Standardization Cluster 3 & Activities \\
\hline Open Networking Foundation (ONF) & $\begin{array}{l}\text { WMWG; Mobile \& Wireless; Optical Transport; Carrier Grade SDN; Migra- } \\
\text { tion; L4-L7; North Bound Interfaces }\end{array}$ \\
\hline Internet Engineering Task Force (IETF)/Internet Research & DMM; I2RS; ForCES; NETCONF; SFC; PCE; ACTN; VNFPool; SDNRG; NVO3; \\
\hline Task Force (IRTF) & NETEXT; INTAREA \\
\hline ETSI & $\begin{array}{l}\text { Network Function Virtualization (NFV) ISG; Mobile Edge Computing (MEC) } \\
\text { ISG }\end{array}$ \\
\hline IEEE 802 & $802.1 C F ; 802.21$ \\
\hline
\end{tabular}

Table 1. Standardization clusters and corresponding relevant activities.

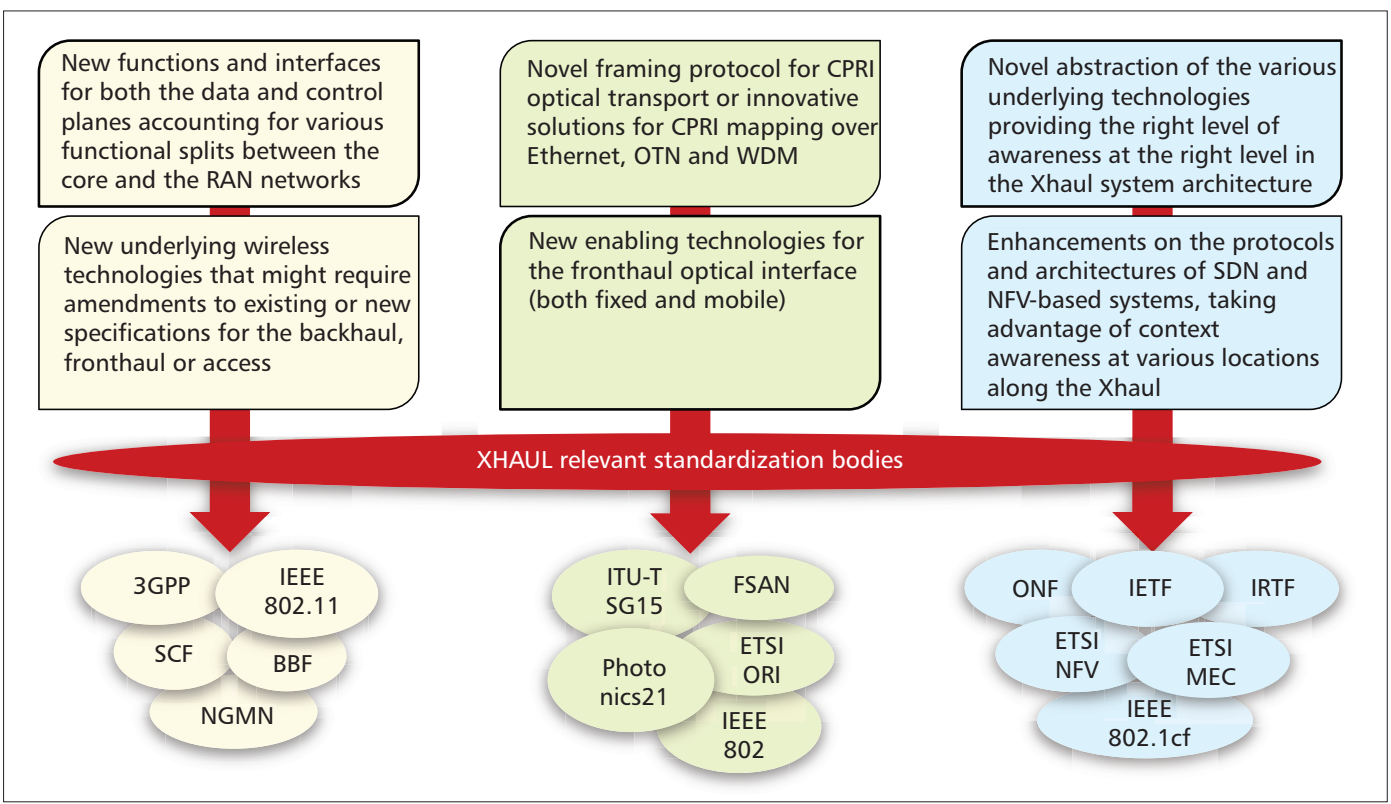

Figure 4. Xhaul architecture impact on standardization bodies.

David Jimenez (Visiona IP), Laurent Bellot (eBlink), Giada Landi (NextWorks), Dragos Vingarzan (Core Network Dynamics), Jose V. Galan (TELNET), Josep Mangues-Bafalluy (CTTC), Tinku Rasheed (CREATE-NET), Claudio Casetti (Politecnico di Torino), Per Odling (Lunds University), and Fang-Chu Chen (ITRI). This work has been partly supported by the Madrid Regional Government through the TIGRE5-CM program (S2013/ICE-2919) and the EU H2020 Xhaul Project (grant no. 671598).

\section{REFERENCES}

[1] Cisco, "Cisco Visual Networking Index: Forecast and Methodology, 2013-2018," white paper, June 2014 http://www.cisco.com/c/en/us/solutions/collateral/service-provider/ip-ngn-ip-next-generationnetwork/white paper c11-481360.pdf.

[2] D. Astely et al., "LTE Release 12 and Beyond," IEEE Commun. Mag., vol. 51, no. 7, 2013, pp. 154-60.

[3] A. Pizzinat et al.," "Things You Should Know about Fronthaul," IEEE/OSA J. Lightwave Tech., 2015.

[4] "C-RAN: The Road towards Green RAN," China Mobile white paper, v. 2, 2011.

[5] M. Nahas et al., "Base Stations Evolution: Toward 4G Technology," 2012 19th IEEE Int'l. Conf. Telecommun. 2012, pp. 1-6. 
The Xhaul architecture

proposed in this article

is a $5 \mathrm{G}$ integrated

backhaul and fronthaul

transport network

enabling a flexible and

software-defined

reconfiguration of all

networking elements in

a multi-tenant and

service oriented unified

management

environment.
[6] P. Rost et al., "Cloud Technologies for Flexible 5G Radio Access Networks," IEEE Commun. Mag., vol. 52, no. 5, May 2014, pp. 68-76.

[7] 3GPP, "Technical Specification Group Radio Access Network; Evolved Universal Terrestrial Radio Access (EUTRA): Medium Access Control (MAC) Protocol Specification, Release 11," Oct. 2012; http://www.qtc. jp/3GPP/Specs/36321-b10.pdf.

[8] "V6.1 Common Public Radio Interface (CPRI); Interface Specification," July 2014; Ericsson AB, Huawei Technologies Col Ltd, NEC Corporation, Nortel Networks SA and Siemens Networks Gmbh \& Co," Ltd, NEC Corporation, Nortel Networks SA, Siemens Networks GmbH \& Co. KG, Ericsson AB, and Huawei Technologies Co Ltd.

[9] "OBSAI System Specification."

[10] V. Jungnickel, "High-Speed Optical Wireless Communications Technologies," OFC '14, 2014, pp. Th1F-5.

[11] A.-J. Lim et al., "Review of Silicon Photonics Foundry Efforts," IEEE J. Sel. Topics Quantum Electron., vol. 20, no. 4, 2014, pp. 405-16.

[12] "Ericsson Mobility Report," June 2014; http://www. ericsson.com/res/docs/2014/ericsson-mobility-reportjune-2014.pdf

[13] J. Aweya, "Implementing Synchronous Ethernet in Telecommunication Systems," IEEE Commun. Surveys \& Tutorials, vol. 16, no. 2, 2nd qtr. 2014, pp. 1080-113.

\section{BIOGRAPHIES}

ANTONIO DE LA OLIVA (aoliva@it.uc3m.es) received his telecommunications engineering degree in 2004 and his Ph.D. in 2008 from UC3M, where he has been working as an associate professor since then. His current line of research is related to networking in extremely dense networks. He is an active contributor to IEEE 802 where he has served as Vice-Chair of IEEE $802.21 \mathrm{~b}$ and Technical Editor of IEEE $802.21 \mathrm{~d}$. He has also served as a Guest Editor of IEEE Communications Magazine. He has published more than 30 papers on different areas of networking including mobility and wireless networks.

XAVIER Costa PÉRÉz is the technical manager of the Xhaul project and manager of the $5 G$ Networks R\&D Group at NEC Laboratories Europe. At NEC he manages several projects focused on $5 \mathrm{G}$ mobile core, backhaul/fronthaul, and access networks, both internally for products roadmap evolution and externally in EU collaborative projects. His team has received several R\&D awards for successful product transfers and holds over 20 patents. He received both his M.Sc. and Ph.D. degrees in telecommunications from the Polytechnic University of Catalonia.

ARTURO AZCORRA received his M.Sc. degree in telecommunications engineering from the Universidad Politécnica de Madrid (UPM) in 1986 and his Ph.D. from the same university in 1989. In 1993, he obtained an M.B.A. with honors from Instituto de Empresa. He has participated in and directed 49 research and technological development projects, including the European ESPRIT, RACE, ACTS, and IST programs. He has coordinated the CONTENT and E-NEXT European Networks of Excellence, and the CARMEN and Xhaul EU projects. He has served as a Program Committee member for many international conferences, including several editions of IEEE PROMS, IDMS, QofIS, ACM CONEXT, and IEEE INFOCOM. He is the founder of the ACM CONEXT conference series and was the General Chair of its first edition. He has published over 100 scientific papers in books, international magazines, and conferences. In addition to his scientific achievements, he has a relevant track record of research management. He was deputy vice rector of academic infrastructures at University Carlos III of Madrid (UC3M) from 2000 to 2007. He served as Director General for Technology Transfer and Corporate Development at the Spanish Ministry of Science and Innovation from 2009 to 2010, and was then appointed Director General of CDTI (the Spanish agency for industrial research) from 2010 to 2012. He is the founder of the international research center IMDEA Networks, and currently is its director, with a double affiliation as a full professor at UC3M.

ANDREA DI GIGLIO received a Dr. Ing. degree in electronic engineering from the University of Pisa, Italy, and Scuola Santa Anna. He is with Telecom Italia Lab. His fields of research are Internet security and optical networks architecture. He has been involved in several European Projects: the architectural Work Package of the IST Project NOBEL, DISCUS as Work Package leader, and ICT STRONGEST as project coordinator.
FABIO CAVALIERE is with Ericsson, Sweden, where he has been since 2006. As an expert in photonic systems and technologies, he contributes to developing strategy in optical transport and access networks. Before joining Ericsson, he was with Marconi, United Kingdom, from 1998 to 2006 in the Photonic Network and System Design Authority. He is coeditor of ITU-T Recommendation G.metro and a member of the Board of Stakeholders of Photonics 21. He has authored several publications and patents on optical communications.

DIRK TIEGELBEKKERS is a senior system specialist in the transport system architecture group for LTE. He has around 18 years of experience in transport network protocols for mobile wireless communication systems, mainly IP-based backhaul networks. He has actively contributed in 2005 to the Nokia specification team for 3.9G by analyzing the performance of MAC/ RLC proposals. In 2008, he was highly involved in the specification of Nokia's Packet Abis solution, which enables operators to employ packet switched networks as a means to provide the transport backhaul for network traffic. He served as technical manager and project lead for several R\&D projects for Nokia Research Centre and Nokia Networks. His current focus area is LTE-A and its impact on mobile transport.

JOHANNES LESSMANN is a senior researcher in the Mobile and Wireless Networks group of NEC Laboratories Europe, Heidelberg, Germany. He currently serves as project manager for internal as well as customer and EU R\&D projects in the domain of mobile backhaul and core networks. His major research topics include network planning, end-to-end resource and QoS management, SDN for transport networks, and mobile carrier cloud. He has authored many standards contributions, and journal and conference publications.

THOMAS HAUSTEIN received his Dr.-Ing. (Ph.D.) degree in mobile communications from the University of Technology Berlin, Germany, in 2006. In 1997, he was with the Fraunhofer Institute for Telecommunications, Heinrich Hertz Institute $(\mathrm{HHI})$, Berlin, where he worked on wireless infrared systems and radio communications with multiple antennas and OFDM. He focused on real-time algorithms for baseband processing and advanced multiuser resource allocation. From 2006 to 2008, he was with Nokia Siemens Networks, where he conducted research for 4G. Since 2009 he has headed the Wireless Communications Department at Fraunhofer $\mathrm{HHI}$ focussing on $5 \mathrm{G}$ and Industrial Wireless.

ALAIN MOURAD is currently leading the R\&D of $5 G$ wireless systems at InterDigital Europe Ltd, United Kingdom. Prior to joining InterDigital, he was a principal engineer at Samsung Electronics R\&D, United Kingdom, and previously a senior engineer at Mitsubishi Electric R\&D Centre Europe, France. Throughout his career, he has been active in the research and standardization of recent wireless communication and broadcasting systems (3GPP LTE, WiMAX $16 \mathrm{~m}$, DVB-T2/NGH, ATSC 3.0). He has several inventions and has authored numerous peer-reviewed scientific publications. He received the Inventor of the Year Award from Samsung Electronics R\&D UK in 2012 and 2013.

PAOLA IOVANNA received her degree in electronics engineering from the University of Roma "Tor Vergata" in 1996. From 1995 to 1997 she collaborated with the FUB research center of Rome, working on fiber optic communications and optical networking. From 1997 to 2000 she worked at Telecom Italia, where she was involved in experimentation of new services based on different access technologies (e.g., XDSL, frame relay, optical). In 2000 she joined Ericsson in the Research Department where she dealt with networking and design solutions for packet and optical technology ( GMPLS, MPLS, Ethernet ). From 2009 to 2012 she was responsible for carrying out research projects on packet and optical routing, control plane, and path computation solutions. In the framework of such activities she realized demonstrators and prototypes as well. In 2012 she was responsible for defining and prototyping SDN solutions for multi-domain transport in collaboration with customers. Since 2014 she has lead a research team to define transport networking and control solutions for $5 \mathrm{G}$. She is actively involved in European projects and is on the Technical Program Committees of international conferences like ECOC. She holds more than 40 patents in routing, traffic engineering systems, and PCE solutions for packet-opto networks based on GMPLS, and multidomain SDN transport, fronthaul, and backhaul solutions for $5 G$, and is an author of several tens of publications in either international scientific journals or conferences. 